\title{
ROBUSTNESS ANALYSIS OF NONLINEAR SYSTEMS SUBJECT TO STATE FEEDBACK LINEARIZATION
}

\author{
Eduardo Rath Rohr* \\ eduardo.rohrestudentmail. newcastle.edu.au
}

Luís Fernando Alves Pereira ${ }^{\dagger}$

pereira@ece.ufrgs.br

\author{
Daniel Ferreira Coutinho $\ddagger$ \\ dcoutinho@pucrs.br
}

* School of Electrical Engineering and Computer Science, University of Newcastle, Callaghan, NSW 2308, Australia.

${ }^{\dagger}$ Departamento de Engenharia Elétrica, Universidade Federal do Rio Grande do Sul, Av. Osvaldo Aranha 103, 90035-190, Porto Alegre, RS, Brasil.

${ }^{\ddagger}$ Grupo de Automação e Controle de Sistemas, Programa de Pós-Graduação em Engenharia Elétrica, Pontifícia Universidade Católica do Rio Grande do Sul, Av Ipiranga, 6681, 90619-900, Porto Alegre, RS, Brasil.

\begin{abstract}
This paper presents a methodology to the robust stability analysis of a class of single-input/single-output nonlinear systems subject to state feedback linearization. The proposed approach allows the analysis of systems whose nonlinearities can be represented in the rational (and polynomial) form. Through a suitable system representation, the stability conditions are described in terms of linear matrix inequalities, which is known to have a convex (numerical) solution. The method is illustrated via a numerical example.
\end{abstract}

KEYWORDS: State Feedback Linearization, Robustness in Nonlinear Systems

\section{RESUMO}

Análise de Robustez de Sistemas Não-lineares Sujeitos à Linearização por Realimentação de Estados

$\mathrm{O}$ artigo apresenta uma metodologia para a análise da estabilidade de uma classe de sistemas não-lineares incertos su-

Artigo submetido em 07/01/2009 (Id.: 00938)

Revisado em 27/02/2009, 10/08/2009

Aceito sob recomendação do Editor Associado Prof. Luis Antonio Aguirre jeitos à linearização por realimentação de estados. A abordagem proposta permite permite a análise de sistemas cujas não-linearidades possam ser expressas nas formas polinomial e racional. Utilizando decomposição em soma de quadrados, as condições de estabilidade são descritas em termos de desigualdades matriciais lineares, que possuem solução numérica eficiente. O método é ilustrado com um exemplo numérico.

PALAVRAS-CHAVE: Linearização por Realimentação de Estados, Robustez em Sistemas Não Lineares

\section{INTRODUCTION}

State feedback linearization (SFL) is a widespread approach to deal with nonlinear control systems, since the resulting linear system can be controlled via well established linear control tools, contrasting with the application of linear control methods to the linear approximation of the nonlinear system, which is valid only when the system remains near the equilibrium point and supposing there are no hard nonlinearities in the vicinity of this point.

Basically, SFL is a method to design a control law that exactly cancels the nonlinearities of a system, allowing the im- 
position of a new arbitrary linear dynamics (Isidori, 1995). Moreover, the approach is valid for the whole state-space, whenever the control law is not constrained to actuator saturation. However, the control design conditions require the exact knowledge of the system dynamics. Thus, in the presence of parametric uncertainty, the cancellation of the nonlinearities is not perfect which may lead to a poor closed-loop performance and even instability.

The robust state feedback linearization (RSFL) is still an open problem and many approaches have been proposed in the last decade to handle model uncertainties. For instance, Guillard and Bourles (Guillard and Bourles, 2000) proposed a RSFL in which the nonlinear system dynamics is transformed through exact cancelations in its linear approximation around an operating point. This result is further developed in (Franco et al., 2006), where theoretical conditions for stability and performance based on the McFarlane-Glover $H_{\infty}$ controller are derived. In (Park, 2003), the robustness properties of a SFL control law is analyzed by means of fuzzy models, and, more recently, Hahn, Mönnigmann and Marquardt (Hahn et al., 2008) has proposed a methodology based on bifurcation analysis for tuning a single parameter of a feedback linearizing controller to take model uncertainties into account.

In the last decade, some researchers have employed the Linear Matrix Inequality (LMI) framework to deal with nonlinear control systems. These methodologies differ between each other from the way the Lyapunov stability conditions are translated into a set of LMIs. The sum of squares (SOS) technique uses semi-definite programming for polynomial systems to decompose the Lyapunov inequalities in terms of SOS (Papachristodoulou and Prajna, 2004). In (Chesi et al., 2004), the authors cast the stability conditions through homogeneous polynomials. The LFR technique of (El Ghaoui and Scorletti, 1996) decomposes the system in terms of a linear fractional representation and uses a quadratic Lyapunov function. The slack variable approach introduced in (Trofino, 2000; Coutinho and Fu, 2002) is based on a particular decomposition of the system in terms of a differential-algebraic representation (DAR) and polynomial Lyapunov functions. The main advantage of considering nonlinear decompositions of the system dynamics is that the original system structure is preserved which allows the use of the well-established linear robust control theory (Boyd et al., 1994) to control and filter design (Coutinho et al., 2008; Coutinho et al., 2009). Besides, through a suitable change of variables, a large class of nonlinear systems can be described in the DAR form including systems with rational, polynomial and trigonometric nonlinearities.

In this scenario, this paper presents an approach to the analysis of single-input/single-output (SISO) uncertain nonlinear systems subject to a SFL controller through the DAR decomposition. The proposed methodology allows the SFL designer to determine an estimate of the admissible parameter set such that the closed-loop system remains stable. The class of nonlinear systems supported by this approach includes the linearizable (through state feedback) systems whose output of the transformed system contains rational, polynomial and trigonometric nonlinearities. The sufficient LMI conditions are obtained to determine an estimate of the domain of attraction of an uncertain system subject to a SFL control. In addition, a line search in the parameters space is performed to determine an admissible set of uncertainties. According to the best knowledge of the authors, there are no similar works available in the control literature that provides a methodology to estimate the domain of attraction of uncertain nonlinear systems subjected to a SFL control based on the LMI framework. Although, similar approaches dealing with saturating control laws such as (Silva Jr et al., 2004; Coutinho et al., 2004; Coutinho and Silva Jr, 2007) can be extended to cope with this problem.

The rest of the paper is organized as follows. Section II states the problem of concern, showing necessary conditions for applying the method. Section III presents the methodology and LMI conditions for stability analysis. A numerical example is presented in Section IV, while Section V ends the paper.

Notation. $\Re^{n}$ denotes the $n$-dimensional Euclidean space, $\Re^{n \times m}$ is the set of $n \times m$ real matrices, $0_{n}$ and $0_{m \times n}$ are the $n \times n$ and $m \times n$ matrices of zeros; $I_{n}$ is the $n \times n$ identity matrix. For a real matrix $S, S^{\prime}$ denotes its transpose and $S>0(S<0)$ means that $S$ is symmetric and positivedefinite (negative-definite). Standard Lie derivative notation is applied throughout the paper.

\section{PROBLEM STATEMENT}

Consider the following nonlinear system

$$
\left\{\begin{array}{l}
\dot{x}=\tilde{f}(x, \delta)+\tilde{g}(x, \delta) u(x) \\
y=h(x)
\end{array}\right.
$$

where $x \in X \in \Re^{n}$ is the state vector, $\delta=\left[\delta_{1}, \delta_{2}, \ldots, \delta_{n_{\delta}}\right]$ is a vector of uncertain parameters supposed to be bounded by the polytope $\Delta \in \Re^{n_{\delta}}, u(x): \Re^{n} \mapsto \Re$ is control signal and $y \in \Re$ is the output signal. Consider that $\tilde{f}(x, \delta): X \times \Delta \mapsto \Re^{n}, \tilde{g}(x, \delta): X \times \Delta \mapsto \Re^{n}$ and $h(x): X \mapsto \Re$ have continuous and well defined partial derivatives in a domain $X \times \Delta$. If we assume $\tilde{f}(x, \delta), \tilde{g}(x, \delta)$ and $h(x)$ have no singularities on the domain of study, there exists a diffeomorphism $T(x)$ proper to the synthesis of the feedback linearization law (Khalil, 2002).

This paper aims to devise a numerical tractable technique to 
analyze the stability of the above uncertain system for all $x \in$ $X$ and $\delta \in \Delta$. To this end, we assume that $X$ is a polytopic set with known vertices. In addition, we suppose that system (1) can be represented in the following differential-algebraic form

$$
\left\{\begin{array}{l}
\dot{x}=A_{1}(x, \delta) x+A_{2}(x, \delta) \pi+A_{3}(x, \delta) u(x) \\
0=\Omega_{1}(x, \delta) x+\Omega_{2}(x, \delta) \pi+\Omega_{3}(x, \delta) u(x)
\end{array}\right.
$$

where $\pi \in \Re^{n_{\pi}}$ is an auxiliary vector containing all functions of $(x, \delta)$ which are not affine on $(x, \delta)$ and $A_{1} \in$ $\Re^{n \times n}, A_{2} \in \Re^{n \times n_{\pi}}, A_{3} \in \Re^{n}, \Omega_{1} \in \Re^{n_{\pi} \times n}, \Omega_{2} \in$ $\Re^{n_{\pi} \times n_{\pi}}$ and $\Omega_{3} \in \Re^{n_{\pi}}$ are affine matrix functions of $(x, \delta)$.

The above representation can model the whole class of polynomial and nonsingular rational functions. The differentialalgebraic representation is called well-posed if the original representation (1) can be recovered by eliminating the auxiliary vector $\pi$ from (2) through the following expression

$$
\pi=-\Omega_{2}^{-1}\left(\Omega_{1} x+\Omega_{3} u(x)\right) .
$$

The well-posedness is guaranteed if the matrix $\Omega_{2}$ is nonsingular, i.e. if $\operatorname{rank}\left(\Omega_{2}(x, \delta)\right)=n, \forall(x, \delta) \in X \times \Delta$.

Through the tangent half-angle formulae, trigonometric nonlinearities can be embedded into the representation (2) with no conservatism as applied for instance in (Danès and Bellot, 2006) and (Coutinho and Danes, 2006) for robotic systems. The basic idea is to apply the change of variable

$$
\theta=2 \arctan (r)
$$

leading to rational functions as follows

$$
\begin{aligned}
\sin \theta & =\frac{2 r}{1+r^{2}} \\
\cos \theta & =\frac{1-r^{2}}{1+r^{2}} \\
\tan \theta & =\frac{2 r}{1-r^{2}} .
\end{aligned}
$$

In other words, trigonometric functions on the variable $\theta$ can be transformed into rational ones in the variable $r$. More details are given in the numerical example later in this paper.

Suppose a SFL control law is designed according to the nominal model, that is: $\delta_{i}=0, i=1, \ldots, n_{\delta}$. When this control law is applied to the uncertain system (1), for any $\delta_{i} \neq 0, i=1, \ldots, n_{\delta}$, the nonlinearities are not exactly canceled and the stability conditions hold only in a neighborhood of the origin.

Similarly to the system representation in (2), the control law can be represented by

$$
\left\{\begin{array}{l}
u(x)=B_{1}(x) x+B_{2}(x) w \\
0=\zeta_{1}(x) x+\zeta_{2}(x) w
\end{array}\right.
$$

where $w \in \Re^{n_{w}}$ contains all functions of $x$ which are not affine in $x$ and $B_{1}(x) \in \Re^{n \times n}, B_{2}(x) \in \Re^{n \times n_{w}}, \zeta_{1}(x) \in$ $\Re^{n_{w} \times n}$ e $\zeta_{2}(x) \in \Re^{n_{w} \times n_{w}}$ are affine matrix functions of $x$. Similarly to the previous case, in order to the vector

$$
w=-\zeta_{2}^{-1} \zeta_{1} x
$$

be well-defined, the matrix $\zeta_{2}$ is assumed to have full rank for all $x$ of interest. Notice that the SFL control law of a rational system is also rational, which means that the control law can be represented in the above manner.

The problem aimed in this paper can be summarized by finding a polytopic set of uncertainties $\Delta$ through a numerically tractable technique, given a system represented by (2), a control law by (5) and a set of admissible states $X$.

\section{STABILITY ANALYSIS}

In this section, we develop stability conditions to the robust analysis of closed-loop nonlinear systems via SFL, where the analysis conditions are cast in terms of LMIs. To this end, the following basic result from the Lyapunov Theory is considered (Kiyama and Iwasaki, 2000).

Lemma 1 Consider the nonlinear system $\dot{x}=$ $f(x(t), \delta(t))+g(x(t), \delta(t)) u(x(t))$ where $f, g: X \times \Delta \mapsto$ $\Re^{n}$ and $u: X \mapsto \Re$ are locally Lipschitz functions such that $f(0, \delta)+g(0, \delta) u(0)=0$. Suppose there exist positive scalars $\epsilon_{1}, \epsilon_{2}$ and $\epsilon_{3}$ and a continuously differentiable function $V: X \mapsto \Re$ that satisfies the following conditions:

$$
\begin{gathered}
\epsilon_{1} x^{\prime} x \leq V(x) \leq \epsilon_{2} x^{\prime} x, \forall x \in X \\
\dot{V}(x) \leq-\epsilon_{3} x^{\prime} x, \forall x \in X \\
D:=\{x: V(x) \leq 1\} \subset X .
\end{gathered}
$$

Then $V(x)$ is a Lyapunov function in D. Moreover, for all $x(0) \in D$ and $\delta(0) \in \Delta$, the trajectory of $x(t)$ approaches the origin when $t \rightarrow \infty$ for all $\delta(t) \in \Delta$.

Consider a quadratic Lyapunov function

$$
V(x)=x^{\prime} P x, P=P^{\prime}>0
$$

whose time derivative is as below

$$
\dot{V}(x)=\dot{x}^{\prime} P x+x^{\prime} P \dot{x} .
$$

Taking into account the DAR, (11) can be represented on its matrix form

$$
\dot{V}(x)=\left[\begin{array}{c}
x \\
\pi \\
u
\end{array}\right]^{\prime}\left[\begin{array}{ccc}
A_{1}^{\prime} P+P A_{1} & P A_{2} & P A_{3} \\
A_{2}^{\prime} P & 0 & 0 \\
A_{3}^{\prime} P & 0 & 0
\end{array}\right]\left[\begin{array}{l}
x \\
\pi \\
u
\end{array}\right] .
$$


Notice that the vector $\left[x^{\prime} \pi^{\prime} u^{\prime}\right]^{\prime}$ is not completely free, due to the relations between the variables $x, \pi$ and $u$. Such relations, expressed in (2), (5), and (6),can be summarized on the following equality constraint.

$$
\left[\begin{array}{cccc}
\Omega_{1} & \Omega_{2} & \Omega_{3} & 0 \\
B_{1} & 0 & -1 & B_{2} \\
\zeta_{1} & 0 & 0 & \zeta_{2}
\end{array}\right]\left[\begin{array}{l}
x \\
\pi \\
u \\
w
\end{array}\right]=0
$$

To handle the above constrained inequality, we can apply the following version of the Finsler's lemma (Oliveira, 2004):

Lemma 2 Given matrices $F_{i}=F_{i}^{\prime} \in \mathbb{R}^{n \times n}$ and $M_{i} \in$ $\mathbb{R}^{m \times n}, i=1, \ldots, r$, then

$$
\lambda^{\prime} F_{i} \lambda>0, \forall \lambda \in \mathbb{R}^{n}: M_{i} \lambda=0, \lambda \neq 0, i=1, \ldots, r
$$

if there exists a matrix $L \in \mathbb{R}^{n \times m}$ such that

$$
F_{i}+L M_{i}+\left(L M_{i}\right)^{\prime}>0, i=1, \ldots, r .
$$

Applying the above lemma, we get the following sufficient condition for (12) subject to (13) to hold

$$
\begin{gathered}
\Upsilon(x, \delta)+L \Xi(x, \delta)+(L \Xi(x, \delta))^{\prime}<0, \\
\forall(x, \delta) \in X \times \Delta
\end{gathered}
$$

where

$$
\begin{aligned}
& \Upsilon(x, \delta)=\left[\begin{array}{cccc}
A_{1}^{\prime} P+P A_{1} & P A_{2} & P A_{3} & 0 \\
A_{2}^{\prime} P & 0 & 0 & 0 \\
A_{3}^{\prime} P & 0 & 0 & 0 \\
0 & 0 & 0 & 0
\end{array}\right], \\
& \Xi(x, \delta)=\left[\begin{array}{cccc}
\Omega_{1} & \Omega_{2} & \Omega_{3} & 0 \\
B_{1} & 0 & -1 & B_{2} \\
\zeta_{1} & 0 & 0 & \zeta_{2}
\end{array}\right] .
\end{aligned}
$$

As the matrix inequality in (14) is affine in $(x, \delta)$, it can be tested only in a finite number of points. More precisely, at the vertices of the polytope $X \times \Delta$.

Before presenting the next lemma, let us introduce two alternative representations of (convex) polytopes. A polytope can be defined in terms of a convex hull of its vertices or, alternatively, by the (closed) intersection of hyperplanes. To illustrate this point, let $X$ be a given polytope in $\Re^{2}$ whose vertices are defined by $\mathcal{V}:=\left\{v_{1}, v_{2}, v_{3}, v_{4}\right\}$, where

$$
v_{1}=\left[\begin{array}{l}
2 \\
3
\end{array}\right], v_{2}=\left[\begin{array}{c}
-2 \\
3
\end{array}\right], v_{3}=\left[\begin{array}{l}
-2 \\
-3
\end{array}\right], v_{4}=\left[\begin{array}{c}
2 \\
-3
\end{array}\right]
$$

A vertex representation of $X$ is defined as the convex hull of $v_{1}, \ldots, v_{4}$, i.e., $X=\operatorname{Co}\left\{v_{1}, \ldots, v_{4}\right\}$. Equivalently, we can define $X$ as follows

$$
X:=\left\{x: a_{k} x \leq 1, k=1, \ldots, 4\right\}
$$

Notice that each inequality defines an hyperplane $\left\{x: a_{k} x \leq\right.$ $1\}$ for which two adjacent $v_{i}$ and $v_{j}$ belongs to its edge, that is:

$$
\begin{aligned}
& v_{1}, v_{2} \in\left\{x: a_{1} x=1\right\}, \\
& v_{2}, v_{3} \in\left\{x: a_{2} x=1\right\}, \\
& v_{3}, v_{4} \in\left\{x: a_{3} x=1\right\}, \\
& v_{4}, v_{1} \in\left\{x: a_{4} x=1\right\} .
\end{aligned}
$$

For a given set $\mathcal{V}$, we can determine the row vectors $a_{k}$ solving the following set of linear systems:

$$
\begin{aligned}
& \left\{\begin{array} { l } 
{ a _ { 1 } v _ { 1 } = 1 } \\
{ a _ { 1 } v _ { 2 } = 1 }
\end{array} \quad \left\{\begin{array}{l}
a_{2} v_{2}=1 \\
a_{2} v_{3}=1
\end{array}\right.\right. \\
& \left\{\begin{array} { l } 
{ a _ { 3 } v _ { 3 } = 1 } \\
{ a _ { 3 } v _ { 4 } = 1 }
\end{array} \quad \left\{\begin{array}{l}
a_{4} v_{4}=1 \\
a_{4} v_{1}=1
\end{array}\right.\right.
\end{aligned}
$$

yielding the following row vectors

$$
\begin{aligned}
& a_{1}=\left[\begin{array}{cc}
0 & 0.33
\end{array}\right], \\
& a_{2}=\left[\begin{array}{cc}
-0.5 & 0
\end{array}\right], \\
& a_{3}=\left[\begin{array}{cc}
0 & -0.33
\end{array}\right], \\
& a_{4}=\left[\begin{array}{cc}
0.5 & 0
\end{array}\right]
\end{aligned}
$$

Lemma 3 provides sufficient conditions to insure that the domain of attraction is bounded to the admissible polytope of states.

Lemma 3 Let $X$ be a given polytope of states defined by its vertices or equivalently by

$$
X:=\left\{x: a_{k} x \leq 1, k=1, \ldots, n_{e}\right\}
$$

where $a_{k} \in \Re^{n}$ are given constant vectors and $n_{e}$ is the number of edges of $X$. The condition $x \in X$ can be written as

$$
2-x^{\prime} a_{k}^{\prime}-a_{k} x \geq 0, k=1, \ldots, n_{e} .
$$

Let the domain $D$ be defined as

$$
D:=\left\{x: x^{\prime} P x \leq 1\right\} .
$$

Thus, if $x \in D$, then

$$
x^{\prime} P x-1 \leq 0
$$

Then, the condition $x \in D \subset X$ is guaranteed if the following holds

$$
1-x^{\prime} a_{k}^{\prime}-a_{k} x+x^{\prime} P x \geq 0, k=1, \ldots, n_{e}
$$

Theorem 4 presents a convex characterization of Lemma 1 .

Theorem 4 Consider system (1) and its DAR as in (2). Consider the SFL control law represented by its SOS as in (5). Let $\Delta$ be a given polytope defining the space of admissible 
uncertainty. Suppose there exist matrices $P=P^{\prime}>0$ and $L$ satisfying the following LMIs at all vertices of $X \times \Delta$ :

$$
\begin{gathered}
\Upsilon(x, \delta)+L \Xi(x, \delta)+(L \Xi(x, \delta))^{\prime}<0 \\
{\left[\begin{array}{rr}
1 & a_{k} \\
a_{k}^{\prime} & P
\end{array}\right] \geq 0, k=1, \ldots, n_{e} .}
\end{gathered}
$$

Then, $V(x)=x^{\prime} P x$ is a Lyapunov function in $X$. Moreover, for all $x(0) \in D$ and $\delta(t) \in \Delta$, the trajectory of $x(t)$ approaches the origin when $t \rightarrow \infty$, where $D:=\{x: V(x) \leq$ $1\}$.

Proof: If the LMIs (24)-(25) are feasible, then by convexity they are also satisfied for all $(x, \delta) \in X \times \Delta$.

Let $\epsilon_{1}$ and $\epsilon_{2}$ be respectively the smallest and largest eigenvalues of $P$. Then, the following holds

$$
\epsilon_{1} x^{\prime} x \leq x^{\prime} P x \leq x^{\prime} x \epsilon_{2} .
$$

Define the vector $\psi:=\left[\begin{array}{llll}x^{\prime} & \pi^{\prime} & u & w^{\prime}\end{array}\right]^{\prime}$. Pre- and postmultiplying the LMI in (24) by $\psi^{\prime}$ and $\psi$ respectively, leads to

$$
\psi^{\prime} \Upsilon \psi+\psi^{\prime} L \Xi \psi+\left(\psi^{\prime} L \Xi \psi\right)^{\prime}<0 .
$$

For simplicity of notation, the affine dependency in $(x, \delta)$ of $\Upsilon$ and $\Xi$ is omitted. Notice that the product $\Xi \psi$ results in a vector of zeros, turning (26) into $\dot{V}(x)=\psi^{\prime} \Upsilon \psi<0$. As the elements of $\Upsilon$ and $\psi$ are bounded, there exists a sufficiently small positive scalar $\epsilon_{3}$ such that

$$
\dot{V}(x) \leq-\epsilon_{3} x^{\prime} x .
$$

Pre- and post-multiplying (25) by $\left[1-x^{\prime}\right]$ and its transpose leads to

$$
\left[\begin{array}{c}
1 \\
-x
\end{array}\right]^{\prime}\left[\begin{array}{cc}
1 & a_{k} \\
a_{k}^{\prime} & P
\end{array}\right]\left[\begin{array}{c}
1 \\
-x
\end{array}\right] \geq 0, k=1, \ldots, n_{e} .
$$

Notice that (27) is the matrix form of (23). The rest of the proof follows straightforwardly from Lemma 1.

Remark 1 Theorem 4 provides an estimate $D$ of the system domain of attraction, but in general we are interested in finding the largest estimate inside $X$. To this end, we may solve the following convex optimization problem

$$
\min _{P, L} \operatorname{trace}(P)
$$

subject to the LMIs in Theorem 4, which corresponds to the minimization of the sum of the squared semi-axis lengths of the ellipsoid $D$.
Remark 2 If we are interested in checking the uncertain domain $\Delta$ in which the closed-loop system is asymptotically stable for a given set of initial conditions $D_{0}=\left\{x: x^{\prime} P_{0} x \leq\right.$ $1\} \subset X$, one can add the condition

$$
P_{0}-P \geq 0
$$

into Theorem 4 to guarantee $D_{0} \subset D$.

\section{NUMERICAL EXAMPLE}

To illustrate the approach, consider the following inverted pendulum equation

$$
\ddot{\theta}=\frac{g \sin (\theta)}{l}-\frac{K \dot{\theta}}{M}+\frac{u}{M l^{2}}
$$

where $g, l, M$ and $K$ are model parameters.

The change of variables (4) is used to transform the trigonometric nonlinearities in rational ones, obtaining the equivalent model

$$
\ddot{r}=\frac{2 r \dot{r}^{2}}{1+r^{2}}+\frac{g r}{l}-\frac{K \dot{r}}{M}+\frac{u\left(1+r^{2}\right)}{2 M l^{2}} .
$$

Defining $x_{1}=r$ and $x_{2}=\dot{r}$, the system (30) can be represented in the state-space form

$$
\begin{aligned}
& \dot{x_{1}}=x_{2} \\
& \dot{x_{2}}=\frac{2 x_{1} x_{2}^{2}}{1+x_{1}^{2}}+\frac{g x_{1}}{l}-\frac{K x_{2}}{M}+\frac{u\left(1+x_{1}^{2}\right)}{2 M l^{2}} . \\
& y=x_{1}
\end{aligned}
$$

The above system is already in the nonlinear controllable form. Thus, the state feedback linearization procedure results in the following control law

$$
\begin{array}{r}
u(x)=\frac{2 M l^{2}}{\left(1+x_{1}^{2}\right)}\left(-k_{1} x_{1}-k_{2} x_{2}+\right. \\
\left.-\frac{2 x_{1} x_{2}^{2}}{1+x_{1}^{2}}-\frac{g x_{1}}{l}+\frac{K x_{2}}{M}\right)
\end{array}
$$

where $k_{1}$ and $k_{2}$ are constant scalars that determine the eigenvalues location in case of exact cancellation of the nonlinear terms.

Let us consider that the uncertain terms $\left(1+\delta_{1}\right)$ and $\left(1+\delta_{2}\right)$ are respectively associated to the parameters $M$ and $K$.

The resulting uncertain system is as follows:

$$
\begin{aligned}
& \dot{x}_{1}=x_{2} \\
& \dot{x}_{2}=\frac{2 x_{1} x_{2}^{2}}{1+x_{1}^{2}}+\frac{g x_{1}}{l}-\frac{K\left(1+\delta_{2}\right) x_{2}}{M\left(1+\delta_{1}\right)}+\frac{u\left(1+x_{1}^{2}\right)}{2 M\left(1+\delta_{1}\right) l^{2}} \\
& y=x_{1}
\end{aligned}
$$


The system (33) can be represented in its differentialalgebraic by defining

$$
\pi=\left[\begin{array}{llllll}
\frac{x_{1} x_{2}}{\left(1+x_{1}^{2}\right)} & \frac{x_{1}}{\left(1+x_{1}^{2}\right)} & \frac{x_{1}^{2}}{\left(1+x_{1}^{2}\right)} & \frac{x_{2}}{\left(1+\delta_{1}\right)} & \frac{u}{\left(1+\delta_{1}\right)} & \frac{x_{1} u}{\left(1+\delta_{1}\right)}
\end{array}\right]^{\prime} .
$$

which leads to the following matrices:

$$
\begin{aligned}
& A_{1}=\left[\begin{array}{cc}
0 & 1 \\
g / l & 0
\end{array}\right], A_{3}=\left[\begin{array}{l}
0 \\
0
\end{array}\right] \\
& A_{2}=\left[\begin{array}{cccccc}
0 & 0 & 0 & 0 & 0 & 0 \\
2 x_{2} & 0 & 0 & \frac{-K\left(1+\delta_{2}\right)}{M} & \frac{1}{2 M l^{2}} & \frac{x_{1}}{2 M l^{2}}
\end{array}\right] \text {, } \\
& \Omega_{1}=\left[\begin{array}{ll}
0 & 0 \\
1 & 0 \\
0 & 0 \\
0 & 1 \\
0 & 0 \\
0 & 0
\end{array}\right], \Omega_{3}=\left[\begin{array}{c}
0 \\
0 \\
0 \\
0 \\
1 \\
x_{1}
\end{array}\right] \\
& \Omega_{2}=\left[\begin{array}{cccccc}
-1 & x_{2} & 0 & 0 & 0 & 0 \\
0 & -1 & -x_{1} & 0 & 0 & 0 \\
0 & x_{1} & -1 & 0 & 0 & 0 \\
0 & 0 & 0 & -\left(1+\delta_{1}\right) & 0 & 0 \\
0 & 0 & 0 & 0 & -\left(1+\delta_{1}\right) & 0 \\
0 & 0 & 0 & 0 & 0 & -\left(1+\delta_{1}\right)
\end{array}\right]
\end{aligned}
$$

Similarly, the SFL control law as presented in (32) can be represented as in (5) by taking

$$
w=\left[\begin{array}{llllll}
\frac{x_{1}}{\left(1+x_{1}^{2}\right)} & \frac{x_{1}^{2}}{\left(1+x_{1}^{2}\right)} & \frac{x_{2}}{\left(1+x_{1}^{2}\right)} & \frac{x_{1} x_{2}}{\left(1+x_{1}^{2}\right)^{2}} & \frac{x_{1}}{\left(1+x_{1}^{2}\right)^{2}} & \frac{x_{1}^{2}}{\left(1+x_{1}^{2}\right)^{2}}
\end{array}\right]^{\prime}
$$

and

$$
\begin{gathered}
B_{1}=\left[\begin{array}{l}
0 \\
0
\end{array}\right]^{\prime}, \quad B_{2}=\left[\begin{array}{c}
2 M l^{2}\left(k_{1}-g / l\right) \\
0 \\
2 M l^{2}\left(k_{2}+K / M\right) \\
-4 x_{2} M l^{2} \\
0 \\
0
\end{array}\right]^{\prime}, \\
\zeta_{1}=\left[\begin{array}{ll}
1 & 0 \\
0 & 0 \\
0 & 1 \\
0 & 0 \\
0 & 0 \\
0 & 0
\end{array}\right], \quad \zeta_{2}=\left[\begin{array}{cccccc}
-1 & -x_{1} & 0 & 0 & 0 & 0 \\
x_{1} & -1 & 0 & 0 & 0 & 0 \\
0 & -x_{2} & -1 & 0 & 0 & 0 \\
0 & 0 & 0 & -1 & x_{2} & 0 \\
1 & 0 & 0 & 0 & -1 & -x_{1} \\
0 & 0 & 0 & 0 & x_{1} & -1
\end{array}\right]
\end{gathered}
$$

For illustrative purposes, in this example we consider the following bounds on the states $x_{1}$ and $x_{2}$ :

$$
\left|x_{1}\right| \leq 0.15,\left|x_{2}\right| \leq 0.15
$$

and the parameters considered in the numerical experiments are given in Table 1.

Solving Theorem 4 (taking the Remark 1 into account) and performing a griding search in the uncertainties $\delta_{1}$ and $\delta_{2}$, we have obtained the following bounds on the uncertain parameters

$$
\left|\delta_{1}\right| \leq 0.097 \text { and }\left|\delta_{2}\right| \leq 0.99
$$

and the following estimate of the domain of attraction

$$
D=\left\{x: x^{\prime}\left[\begin{array}{ll}
62 & 33 \\
33 & 64
\end{array}\right] x \leq 1\right\}
$$

Table 1: Pendulum Parameters

\begin{tabular}{|c|c|}
\hline Parameter & Value \\
\hline$M$ & $2 \mathrm{~kg}$ \\
\hline$l$ & $1 \mathrm{~m}$ \\
\hline$g$ & $9.8 \mathrm{~m} / s^{2}$ \\
\hline$K$ & $0.5 \mathrm{~N} . \mathrm{s} / \mathrm{m}$ \\
\hline$k_{1}$ & -1 \\
\hline$k_{2}$ & -2 \\
\hline
\end{tabular}

To check the accuracy of these results, several simulations have been performed for a set of initial conditions satisfying $V(0)=1$ and at the extremum values of $\delta_{1}$ and $\delta_{2}$ leading to four trajectories for each initial condition. Figure 1 shows the polytope of admissible states $X$, the estimate of the domain of attraction $D$ and the stable system trajectories (obtained through simulations).

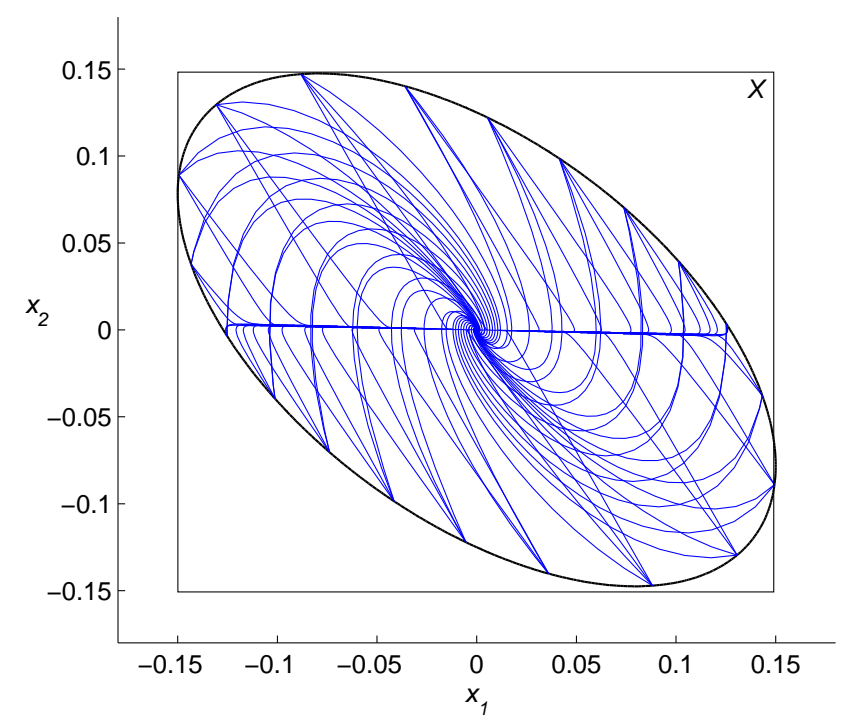

Figure 1: Domain of Attraction and Phase Trajectories.

We have also made an analysis of conservativeness by increasing the bound on $\delta_{1}$ and testing the system trajectories if they converge or not to the equilibrium point. We have noted that for $\delta_{1} \geq 0.102$ the system trajectories do not converge to the equilibrium point under analysis as illustrate in Figure 2, where the red dotted lines represent unstable trajectories. It turns out this value is only $5 \%$ larger than the estimated bound on $\delta_{1}$ demonstrating that the method is not conservative for this particular system. 


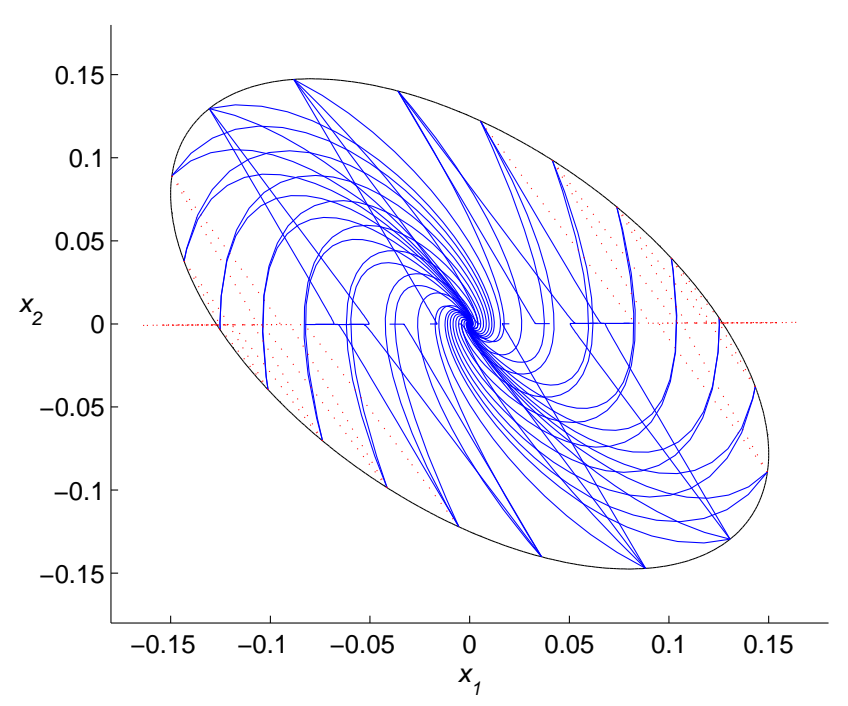

Figure 2: Phase trajectories for $\delta_{1}=0.102$.

\section{CONCLUSIONS}

The paper has presented a methodology to the stability analysis of uncertain nonlinear systems subject to a state feedback linearization control law. Through a suitable system and control law decompositions, we devise LMI conditions that ensure the local asymptotical stability of the uncertain system while providing an estimate of the system domain of attraction based on quadratic Lyapunov functions. In addition, we can estimate the set of admissible uncertainties via a line search on the parameter bounds. The approach have been applied to the stability analysis of an inverted pendulum system subject to a stabilizing feedback linearization control demonstrating the applicability of the proposed results. We emphasize the results derived in this paper can be easily extended to deal with multi-input multi-output feedback linearizing schemes, and, currently, the authors are extending the methodology to deal with actuator saturation.

\section{REFERENCES}

Boyd, S., El Ghaoui, L., Feron, E. and Balakrishnan, V. (1994). Linear Matrix Inequalities in System and Control Theory, SIAM.

Chesi, G., Garulli, A., Tesi, A. and Vicino, A. (2004). Robust analysis of LFR systems through homogeneous polynomial Lyapunov functions, IEEE Transactions on Automatic Control 49(7): 1211-1215.

Coutinho, D. and Danes, P. (2006). Piecewise Lyapunov functions to the stability analysis of rational systems subject to multiple state constraints, Proc. 45th IEEE Conference on Decision and Control, pp. 5801-5806.
Coutinho, D. and Fu, M. (2002). Guaranteed cost control of uncertain nonlinear systems via polynomial lyapunov functions, IEEE Transactions on Automatic Control 47(9): 1575-1580.

Coutinho, D., Fu, M., Trofino, A. and Danes, P. (2008). L $2^{-}$ gain analysis and control of uncertain nonlinear systems with bounded disturbance inputs, International Journal of Robust and Nonlinear Control 18(1): 88110.

Coutinho, D., Pagano, D. and Trofino, A. (2004). On the estimation of robust stability regions for nonlinear systems with saturation, Controle \& Automação 15: 269-278.

Coutinho, D. and Silva Jr, J. d. (2007). Estimating the Region of Attraction of Nonlinear Control Systems with Saturating Actuators, Proc. American Control Conference, pp. 4715-4720.

Coutinho, D., Souza, C., Barbosa, K. and Trofino, A. (2009). Robust linear $\mathrm{H}_{\infty}$ filter design for a class of uncertain nonlinear systems: an LMI approach, SIAM Journal on Control and Optimization 48(3): 1452-1472.

Danès, P. and Bellot, D. (2006). Towards an LMI approach to multicriteria visual servoing in robotics, European Journal of Control 12(1): 86-110.

El Ghaoui, L. and Scorletti, G. (1996). Control of rational systems using linear-fractional representations and linear matrix inequalities, Automatica 32(9): 1273-1284.

Franco, A., Bourles, H., De Pieri, E. and Guillard, H. (2006). Robust nonlinear control associating robust feedback linearization and $H_{\infty}$ control, IEEE Transactions on Automatic Control 51(7): 1200-1207.

Guillard, H. and Bourles, H. (2000). Robust feedback linearization, Proc. 14 th International Symposium on Mathematical Theory of Networks and Systems .

Hahn, J., Mönnigmann, M. and Marquardt, W. (2008). On the use of bifurcation analysis for robust controller tuning for nonlinear systems, Journal of Process Control 18(3-4): 408-420.

Isidori, A. (1995). Nonlinear Control Systems, Springer.

Khalil, H. (2002). Nonlinear systems, Prentice Hall, Upper Saddle River.

Kiyama, T. and Iwasaki, T. (2000). On the use of multi-loop circle criterion for saturating control synthesis, Systems \& Control Letters, Vol. 41, pp. 105-114.

Oliveira, M. (2004). Novos testes de estabilidade para sistemas lineares, Controle \& Automação 15: 17-23. 
Papachristodoulou, A. and Prajna, S. (2004). Analysis of non-polynomial systems using the sum of squares decomposition, Positive Polynomials in Control, Springer

Park, C. (2003). LMI-based robust stability analysis for fuzzy feedback linearization regulators with its applications, Information Sciences 152: 287-301.

Silva Jr, J. d., Tarbouriech, S. and Reginatto (2004). Application of hybrid and polytopic modelling to the stability analysis of linear systems with saturating inputs, Controle \& Automação 15: 401-412.

Trofino, A. (2000). Robust stability and domain of attraction of uncertain nonlinear systems, Proc. American Control Conference $\mathbf{5}$. 